\title{
KOGNITIV-SEMANTISCHE SPEZIFIK VON VERKAUFSGESPRÄCHEN: PHRASEOLOGISCHE FÜGUNGEN UND FUNKTIONSVERBGEFÜGE
}

Dieser Beitrag ist ein Überblick über kognitive Mechanismen und konzentriert sich vor allem auf kognitive Möglichkeiten der Untersuchung mentaler Prozesse bei Erwerb und Verwendung der Sprache.

Die Kognition, eine auf Erkenntnis orientierte Wissenschaft, entwickelt sich in verschiedenen Bereichen des Wissens. „Die Kognitive Wissenschaft ist mit dem Anspruch angetreten, nicht nur einige Teilprozesse, sondern den menschlichen Geist in seiner realen Komplexheit zu untersuchen" (Rickheit, Strohner 1993: 5). Sie empfiehlt nicht nur Modelle und Strukturen zur Beschreibung der Funktionsweise des menschlichen Geistes, sondern sie unterstützt auch die experimentelle Untersuchung und Entwicklung dieser Modelle.

Auch in der Sprachwissenschaft hat die Kognition ihren Ausdruck gefunden und verursachte eine kognitive Wende in der Linguistik, indem sie die Erforschung der Sprache bereicherte und neue Forschungsaspekte einführte. Die Kognitive Linguistik weist auf die Möglichkeit vom Eindringen in die Erkenntnisprozesse des Menschen, in die Natur der im Geist bestehenden Begriffe hin, denn diese bestimmen unsere Perzeption der Welt näher.

Die Zielsetzung der Kognitiven Linguistik ist eine adäquate Charakterisierung der Struktur und Organisation vom sprachlichen Wissen als Teil der menschlichen Erkenntnis (Kognition). Das sprachliche Wissen wird also als reale psychologische Strukturen verstanden, die die sprachliche Fähigkeit eines Sprechers ausmachen. Es geht hier um solche sprachlichen Fähigkeiten wie z.B.: Verständnis, Auffassungsvermögen der sprachlichen Konventionen. Die Kognitive Linguistik geht davon aus, dass die Grammatik einer Sprache ein strukturiertes Inventar von konventionellen Einheiten ist, das einem Sprecher zur Verfügung steht.

Eine Einheit (unit) ist ein „Programm“, das ohne kognitiven Aufwand sofort ablaufen kann (Langacker 1991: 15). Eine Struktur ergibt sich daraus, dass bestimmte 
Einheiten Teile einer höheren Einheit sein können; so wird z.B. ein Dativaffix nur die Komponente eines Wortes sein und ein (flektiertes) Wort ist meistens die Komponente eines Satzes, usw.

Die Kognitive Grammatik konzentriert sich vor allem auf die Darstellung der syntaktischen Grundmuster, die dann jeweils mit lexikalischem Material gefüllt werden. Jede syntaktische Konstruktion verfügt demnach über eine eigenständige Bedeutung, zu der die jeweilige Verbbedeutung und die Bedeutungen der Verbargumente hinzutreten.

Nach Langacker (1991) wird der Prozess der Zusammenfassung von simplen Konzepten zu komplexen Konzepten durch die Möglichkeiten der Metaphorisierung verwirklicht. Jede Zeichenverwendung hat als Grundlage die Metapher. Wenn man sich mit der Analyse der Verkaufsgespräche befasst, muss man unbedingt die besondere Rolle der Gesten betrachten, die in der Kognitiven Linguistik einen integralen Teil der Sprache bilden. Gesten und Sprache bilden genauso wie Worte, Phrasen und Sätze ein Sprachsystem. Sie stellen genau so gut wie Begriffe Gedankenprozesse des Menschen dar (McNeil 1992).

Gesten können Worte ersetzen und Begriffe (auch abstrakte) ausdrücken. Sprache und Gesten bilden zusammen eine Kommunikation und auf der Ebene der Kommunikation ergänzen sie sich.

Die Kognitive Linguistik zieht künstlichen Grenzen zwischen Sprache, Perzeption, Konzeption. Alle genannten Aspekte wirken zusammen auf den Prozess der Kommunikation.

Man kann beobachten, dass Gestikulation vielfältigere und kompliziertere sprachliche Gedankenstrukturen wiedergibt als die lineare Sprache. Die Gesten übermitteln auch das, was in der verbalen Kommunikation nicht ausgedrückt wird, denn sie weisen nicht nur auf Bilderreichtum des ausgesprochenen Gedankens hin, sondern drücken auch Emotionen, Verhältnis zum Hörer, Metastruktur des Textes aus.

Gesten finden auch einen sehr wichtigen Ausdruck bei der Verkaufskommunikation, die Gestikulation mit Händen, Kopf und Körper soll in diesem Sprachbereich die Bedeutung von Worten und Aussagen betonen.

McNeil (1992) unterscheidet fünf grundsätzliche Typen von Gesten:

- Deiktische Gesten - (auch Deixeme genannt) - die Deixeme können auf die Situation durch Demonstrativpronomen, Zeit- und Ortsadverbien verweisen, aber auch auf die Redekostellation selbst (anaphorisch) (vgl. dazu auch die früheren Ausführungen in Heupel 1973: 43f.). Deiktische Gesten beziehen sich auf außersprachliche Elemente in Relation zur jeweiligen Sprechsituation. Sie erscheinen oft in Situationen, in denen keine konkreten, realen Elemente aufzuweisen sind. Sie beziehen sich also auf den mentalen Raum der Äußerungssituation, der dann vom Geist des Sprechers zum realen Raum gebracht wird. Das alles, was im Geist des Sprechers entsteht, wird dann vor dem Hörer in seinem realen Raum aufgezeigt. 
- Schläge (beats) - Gesten, die besonders mit der Aussage verbunden sind, der Sprecher will damit die Bedeutung seiner Aussage betonen. Kurze Handbewegungen bringen wesentliche Informationen zum Ausdruck, zeigen das Verhältnis des Sprechers zum Hörer.

- Kohäsionsgesten - Nach J. Lyons (1972) versteht man unter Kohäsion den inneren Zusammenhalt der Wörter und Morpheme. McNeil (1992) bezeichnet Kohäsionsgesten auch als Verknüpfungen des Textes. Sie können aus Ikonen, Deixemen, Metaphern, Schlägen bestehen. Der Zusammenhang wird durch Wiederholung derselben Form von Geste und Bewegung gebildet. Die Kohäsionsgeste wird oft vor und nach der Aussage des Sprechers sichtbar. Die Kohäsionsgeste hat zum Ziel, den Hörer zu informieren, wohin er nach der Abschweifung der Aussage zum Thema zurückkehren soll. Die erwähnten Gesten sind eng mit der Aussage verbunden und ohne sie könnten sie nicht stattfinden.

- Ikonische Gesten - sind nach C. Heupel (1973) Zeichen, die Ähnlichkeit mit dem Objekt aufweisen, indem sie Aspekte des realen Objekts abbildhaft imitieren. McNeil (1992) bezeichnet ikonische Gesten als Zeichen von Gegenständen und Geschehnissen. Sie enthalten eine direkte Gemeinsamkeit zwischen Begriffsstruktur und Form der Geste.

- Metaphorische Gesten haben eine ganz andere Bedeutung. Sie sind Zeichen von Geistesprozessen. Metaphorische Gesten drücken abstrakte Idee auf eine abbildbare Art aus.

Die kognitiven Prozesse, die in der Sprachverarbeitung ablaufen, werden durch verschiedene Modelle dargestellt, die unter dem Begriff des Konnektionismus auftreten. „Die konnektionistischen Modelle postulieren statt strukturierter Einheit und strukturabhängiger, serieller Prozesse vernetzte Elemente und parallel ablaufende Informationsverarbeitungsvorgänge. Die Modellierung kognitiver Prozesse ist die generelle Zielsetzung des Konnektionismus, dessen Grundideen durch Forschungsergebnisse der Neurophysiologie des menschlichen Gehirns injiziert werden. Die konnektionistischen (oder auch subsymbolischen) Modelle inkorporieren eine große Anzahl einfacher Einheiten oder Knoten, die miteinander vernetzt sind" (Schwarz 1996: 22f.).

Die Einheiten weisen bestimmte Werte für ihre Aktivierbarkeit auf. Die einzelnen Knoten sind durch erregende oder hemmende Relationen miteinander verknüpft.

Einige der kognitiven Modelle weisen große Ähnlichkeiten zueinander auf, wobei sich nur der Blickwinkel, der gegenüber der Problematik eingenommen wird, leicht verschiebt. Rickheit, Strohner (1993) stellen eine Einteilung von autonomen und interaktiven Modellen dar. Die autonomen Modelle nehmen weniger Bezug auf introspektiv erfassbare Mechanismen der Sprachrezeption als die später entwickelten interaktiven Modelle. Autonome Modelle basieren auf der Annahme, dass die einzelnen kognitiven Subsysteme, die an der Sprachverarbeitung beteiligt sind, unabhängig voneinander arbeiten. 
Das Ziel der Analyse der Verkaufsgespräche besteht auch darin, dass man anhand der kognitiven Mechanismen zur Erkenntnis der versteckten Inhalte kommt, die vom Sprecher (Verkäufer) aufgebaut werden. Die Kognitive Wissenschaft kann Belege dafür liefern, dass die Bedeutung, also der Sinn eines Verkaufstextes, nicht in diesem selbst angelegt ist, sondern sowohl vom Sprecher (Verkäufer) als auch vom Hörer (Kunden) in einem subjektiven, dynamischen Prozess konstruiert werden kann.

Nach dem Postulat der Kognitiven Linguistik gibt es derzeit keine Theorie, die auch nur ansatzweise alle relevanten Aspekte menschlichen Wissens und seiner Verwendung erklären kann.

Zwei Aspekte der kommunikativen Absichten oder Ziele müssen hier unterschieden werden: Handlungstypen und mentale Zustände des Hörers. Um die Beziehung zwischen den beiden Aspekten zu untersuchen, muss man die Situation der Verkaufsgespräche bestimmen. Der mentale Zustand des Hörers, der durch eine Sprachhandlung bewirkt wird, ist eine Reaktion auf den gesamten Inhalt der Äußerung und auf andere schwer kalkulierbare Faktoren der Situation.

Phraseologische Wendungen werden im Allgemeinen in den Verkaufsgesprächen nicht allzu häufig verwendet. Diese Situation entspricht auch vielen anderen Kommunikationsbereichen, in denen Phraseologismen bei weitem nicht so oft vorkommen, wie man hätte annehmen können. Der Grund bei den Verkaufsgesprächen ist evident: Es geht hierbei um Dialoge, bei denen die Intentionen des Verkäufers in der Regel durch andere, viel ökonomischere Sprachmittel erreicht werden können. Der Verkäufer versucht seine Sprache möglichst sachlich zu gestalten und - bis auf wenige Ausnahmen - nicht durch zusätzliche, manchmal schwerfällige, Konstruktionen zu überlasten. Wenn schon feste Wendungen auftreten, sind dies vor allem allgemein übliche Klischees bzw. zum Nominalstil gehörige Funktionsverbgefüge bzw. Streckformen des Verbs, die statt einfacher finiter Verben verwendet werden, um beim Kunden eine Vorstellung von „Solidität“ des Verkäufers zu erwecken. Dieser Effekt der Funktionsverbgefüge beruht darauf, dass sie durch angebliche „Kompliziertheit" ihres Aufbaus indirekt von der guten Ausbildung und dem Fachwissen eines Sprechers zeugen. Wer statt funktionieren die Wendung eine Funktion führen, statt bitten - eine Bitte stellen, statt aufführen - zur Aufführung bringen etc. verwendet, gilt als gebildet, da sich diese und andere Funktionsverbgefüge vor allem in sachlichen Texten und wissenschaftlicher Literatur finden lassen. Wer sie also aktiv in seiner Rede gebraucht, gilt in aller Regel als jemand, der einen fachgerecht beraten kann. Aber auch andere Funktionsverbgefüge, die zur Alltagssprache gehören, können unter bestimmten Kontextbedingungen ähnliche Effekte auslösen, einfach weil sie häufig den Stilregister des Dialogs erhöhen und dadurch das Vertrauen des Kunden zum Verkäufer und seinen Empfehlungen steigern z.B.:

Verkäufer: Welche von den beiden Farben, Frau Huber, würden Sie bevorzugen, die Eierschalenfarbe (A) oder hellbeige (B)?

Kunde: Die Farbe B gefällt mir sehr gut. 
Verkäufer: Ja, ich würde in Ihrem Falle auch diese Farbe in Betracht ziehen, da Sie einen etwas dunklen Raum haben, wird der helle Ton lichtspendend wirken.

Kunde: Das ist eigentlich mehr, als ich vorgesehen hatte.

Verkäufer: Ja, nur dürfen wir nicht vergessen, Frau und Herr Huber, dass dies eine Investition ist, die Ihnen über Jahre hinweg viel Nutzen bringen und Freude bereiten wird. Zudem werden wir Ihnen diese Gruppe nach Haus liefern.

Verkäufer: Haben die Kinder Freude an ihrem Studio und sind Sie zufrieden mit der Qualität?

Verkäufer: Sie werden sicher über Jahre hinweg ihre Freude an diesem Schlafzimmer haben.

Verkäufer: Machen wir doch den Versuch!

Verkäufer: Sie haben die beste Wahl getroffen.

Verkäufer: Sie haben eine gute Wahl getroffen.

Die relativ häufige Verwendung von Funktionsverbgefügen, darunter solchen, die oft wiederholt werden (wie z.B. Freude haben statt sich freuen, Freude bereiten statt freuen, Nutzen bringen statt nutzen/nützlich sein, den Versuch machen statt versuchen, eine Wahl treffen statt wählen etc.), tragen auch allgemein der Tendenz zum Nominalstil Rechnung, die sich gegenwärtig in den Bereichen der gesellschaftlichen Kommunikation durchsetzt, die nicht unbedingt offizielle Beziehungen und amtliche Umgangsformen voraussetzen. Ursprünglich aus der Schriftsprache gekommen, finden sie heutzutage zunehmend in der mündlichen Kommunikation Verbreitung, darunter, wie man aus diesen Ausführungen sehen kann, bei persuasiven Dialogen im Konsumbereich.

Seltener sind verbale Phraseologismen mit übertragener idiomatischer Bedeutung, die einem anderen Zweck dienen, nämlich der Überzeugung durch bildhaftexpressive Rede. Das Bedürfnis nach solchen Mitteln entsteht, vor allem, wenn der Verkaufsgegenstand teure Waren sind, bei denen die Überzeugung des Kunden zu einer Kaufentscheidung nicht leicht fällt z.B.:

Verkäufer: ... wenn Sie laufen, joggen oder mit dem Auto bestimmte Strecken messen wollen, das wäre das mit Chronograf, mit Stoppuhr, zwei Drücker rechts, die einfach in der Handhabung sind, man muss nicht unbedingt technisch dafür begabt sein, kann jeder Laie tragen.

Die phraseologische Einheit Strecken messen, die hier statt fahren verwendet wird, unterstreicht, dass die Autofahrt lange dauert, wobei das Verb messen im Phraseologismus bewusst mit dem Zeitmessen durch die Uhr assoziiert wird, die in diesem Verkaufsgespräch den Verkaufsgegenstand bildet.

In einem anderen Verkaufsgespräch wird die feste verbale Wendung eine Linie ziehen, verstärkt durch das Adjektiv schön, zum Ausdruck des Gedankens verwendet, dass in der zu verkaufenden Polstergruppe alle Teile aufeinander farblich, stilistisch und materialgemäß abgestimmt werden sollen:

Verkäufer: Ich würde zum Beispiel etwas popige Bettwäsche auswählen. Was meinen Sie? Kunde: Das ist eine gute Idee, unser Studio ist auch in Ahornholz, erinnern Sie sich noch? Verkäufer: Ja sicher, so können wir eine schöne Linie ziehen. 
Neben verbalen Phraseologismen werden deren nonverbale Derivate verwendet, d.h. phraseologisch gebundene Wendungen ohne Verbalteil z.B.:

Wissen Sie, was Gott und die Welt anbelangt, hätte ich sowieso viele offene Fragen.

Die Wendung Gott und die Welt ist ein Derivat aus dem verbalen Phraseologismus mit jmdm. über Gott und die Welt reden (können). Diese verbale phraseologische Einheit wird zur Charakterisierung eines Menschen verwendet, der ein sehr guter, offener und toleranter Gesprächspartner ist, mit dem man über alles reden kann, ohne dass er seinem Gesprächspartner etwas übel nimmt, über bestimmte Probleme gar nicht reden will oder immer dogmatisch auf seinem Standpunkt beharrt. Gott und die Welt haben in dieser Wendung heutzutage keine direkte Bedeutung mehr, der Phraseologismus bedeutet nicht, dass man unbedingt über religiöse Fragen etc. redet, über Gott und die Welt hat die phraseologische Bedeutung über alles Mögliche. Daher bedeutet das phraseologische Derivat Gott und die Welt 'alles Mögliche, alles Denkbare'. Aber in dem angeführten Kontext wird die Bedeutung des Phraseologismus durch konkrete Kontextbedingungen „dephraseologisiert“, und die Wendung muss wörtlich interpretiert werden. Diese direkte, also nicht phraseologische, Deutung ist durch die allgemeine Gesprächssituation bedingt. Als Verkaufsgegenstand tritt hier nämlich die Bibel auf. Der Verkäufer überzeugt die Kunden zum Kauf dieses Buches als Geburtstagsgeschenk für ihren Enkel. Als gläubiger Mensch engagiert er sich dabei sehr stark und motiviert seine Kunden durch religiöse Argumente. In seiner Replik gibt der Kunde dem Verkäufer zu wissen, dass ihn religiöse Gründe eigentlich nicht stark überzeugen können. Die feste Wendung Gott und die Welt bekommt in diesem Kontext natürlich seine alte, nicht übertragene Bedeutung, es geht eben nicht um 'alles Mögliche', sondern konkret um Gott. Der zweite Teil der Wendung 'und die Welt' wird gebraucht, damit der Phraseologismus vollständig ist. Der besprochene Beleg ist ein transparentes Beispiel für den Funktionswandel des Phraseologismus bei Erhaltung seiner Form in Zusammenhang mit der Umschaltung der Kommunikation auf einen anderen konzeptuellen Bereich. Es ist zu betonen, dass phraseologische Einheiten gerade bei solchen Umschaltungen ein sehr hohes Potential haben, da sie im Unterschied zu nichtphraseologischen Entitäten sehr häufig die Möglichkeit haben, „dephraseologisiert“ und dadurch in direkter Bedeutung verwendet zu werden.

Aus nichtverbalen Phraseologismen ist auch die im Folgenden verwendete phraseologische Wendung die goldene Mitte zu erwähnen:

Kunde: Jetzt schlafen wir auf etwas härteren Matratzen, für mich ist das OK, meine Frau klagt jedoch beim Aufstehen gelegentlich über Schmerzen im Schulterbereich.

Verkäufer: Um Ihre Frage zu beantworten, was besser ist, würde ich meinen, dass die goldene Mitte in Ihrem Fall die Lösung wäre. 
Dieser metaphorische, nominale Phraseologismus, in dem das abstrakte Substantiv Mitte mit dem metaphorisch verwendeten Adjektiv golden in der Bedeutung 'das Beste' verbunden wird, wird zur optimalen Erreichung des persuasiven Ziels der Verkäuferin eingesetzt.

Insgesamt kann festgestellt werden, dass Phraseologismen verschiedener Art vor allem in längeren Verkaufsgesprächen mit einem relativ teuren Verkaufsgegenstand und daher einem nicht einfach erreichbaren persuasiven Ziel des Verkäufers auftreten. Sie dienen neben emphatischer Intonation, rhetorischen Fragen, Wiederholungen, einem spezifischen Satzbau, der sachlichen Argumentation etc. der Verstärkung des verbalen Einflusses auf den Kunden mit dem Zweck, ihn von seinem Kaufwunsch zu überzeugen bzw. den entstandenen Kaufwunsch zu stärken oder auch einen anderen, für das Geschäft erstrebenswerten Kaufwunsch zu erzielen. Dabei werden vor allem die konzeptuell relevanten Bereiche des gesellschaftlichen Lebens in die Kommunikation einbezogen und durch den Einsatz dieser sprachlichen Mittel mit dem individuellen Leben und den individuellen Präferenzen des Kunden in Verbindung gebracht. Das persuasive Potential des Phraseologismus ist im Allgemeinen hoch, darf aber auch nicht überschätzt werden, wovon relativ wenige Entitäten dieser Art zeugen, die in der Untersuchung ermittelt werden konnten. Da nun diese Analyse auf einem quantitativ repräsentativen und vom Verkaufsgegenstand her ausreichend vielfältigen Material basiert, kann mit sehr hoher Wahrscheinlichkeit angenommen werden, dass die Schlussfolgerungen bezüglich der phraseologischen Fügungen, die hier aufgeführt worden sind, für Verkaufsgespräche generell gültig sind.

\section{Literatur}

Heupel, C. (1973): Taschenwörterbuch der Linguistik. München: List.

Langacker, R.W. (1991): Foundations of cognitive grammar. Stanford, Calif.: Stanford University Press. Lyons, J. (1972): Einführung in die moderne Linguistik. München: Beck.

McNeil, D. (1992): What Gestures Reveal about Thought. The University of Chicago Press.

Rickheit, G., Strohner, H. (1993): Grundlagen der kognitiven Sprachverarbeitung: Modelle, Methoden, Ergebnisse. Tübingen und Basel: A. Francke Verlag.

Schwarz, M. (1996): Einführung in die Kognitive Linguistik. Tübingen und Basel: A. Francke Verlag. 